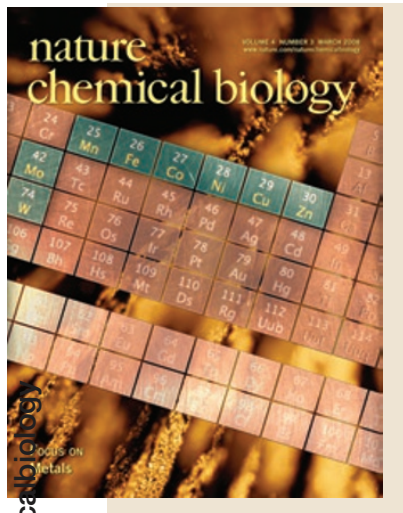

\title{
Focus on metals
}

Metal ions play critical roles in all organisms. While significant progress has been made, for instance in understanding the structural basis of metalloenzyme and metal transport protein function [Elements, p. 157], much still remains to be uncovered about the roles of metal ions in biological systems. In this issue, we highlight some important recent advances in understanding the biology of metals. Cells have elaborate mechanisms for regulating the import, transport and delivery of metal ions. Kim, Nevitt and Thiele review the current understanding of cellular copper homeostasis and highlight the many pathways and mechanisms that remain to be elucidated [Reviews, p. 176]. Davis and O'Halloran highlight the new types of biological coordination chemistries that are being discovered in these copper trafficking proteins [Commentaries, p. 148]. Transition metal cofactors play key roles in catalyzing many enzymatic reactions that involve molecular oxygen as a substrate. Kovaleva and Lipscomb illustrate how non-heme Fe(II) oxidases and oxygenases catalyze oxygen activation by a remarkable variety of chemical mechanisms [Reviews, p. 186]. Loenarz and Schofield describe how this chemical diversity is matched by an equal array of biological processes, from epigenetics to obesity, that have recently been shown to involve this family of enzymes [Commentaries, p. 152]. These pairs of articles on copper trafficking and non-heme Fe(॥) enzymes highlight the exciting advances that are being made in these systems from both the biological and chemical perspectives. Despite this progress, new tools and approaches are needed to gain a better understanding of the roles that metal ions play in cells and in whole organisms. Domaille, Que and Chang highlight recent developments in creating small-molecule fluorescent probes for visualizing metal ions in living cells [Reviews, p. 168]. Thiele and Gitlin argue for a new approach towards a systems-level understanding of how transition metal metabolism functions within whole organisms and ecosystems [Commentaries, p. 145]. We hope this collection of articles highlights the excitement of the chemical biology of metals and the importance of bringing together chemical and biological approaches to address remaining questions in the field.

\section{Malarial parasite escape route}

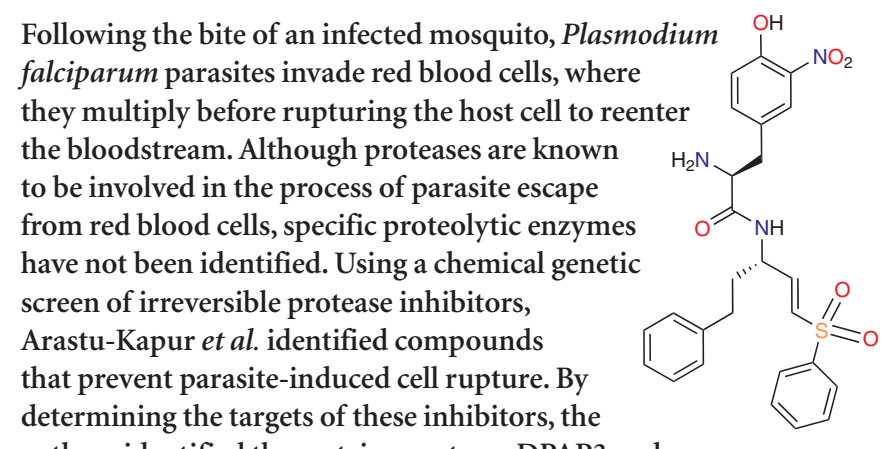
authors identified the cysteine protease DPAP3 and the serine protease PfSUB1 as part of this proteolytic cascade. These results provide a first glimpse at the red blood cell rupture pathway and identify potential new targets for antimalarial therapeutics. [Articles, p. 203; News \& Views, p. 161]

\section{Cobalamin by express delivery}

The adenosylcobalamin-dependent enzyme methylmalonyl coenzyme A mutase (MCM) catalyzes the conversion of methylmalonyl-CoA to succinyl-CoA. Adenosyltransferase (ATR) catalyzes the final step in the formation of the adenosylcobalamin cofactor. Padovani et al. now report that ATR also functions as a chaperone to deliver adenosylcobalamin to MCM. Kinetic and thermodynamic analysis suggests that adenosylcobalamin was reversibly transferred from ATR to MCM without cofactor release into solution. Further mutagenesis studies suggest that an ATR histidine that coordinates adenosylcobalamin plays a role in mediating the transfer. Because free adenosylcobalamin is present at very low concentrations within cells, the combined role of ATR in the final enzymatic step of cofactor biosynthesis and in cofactor delivery ensures that adenosylcobalamin arrives at its destination. [Brief Communications, p. 194; News \& Views, p. 158] $J K$

Written by Catherine Goodman \& Joanne Kotz

\section{Amyloid inhibitors en masse}

Fibrils resulting from protein aggregation in neurodegenerative diseases have served as a common target in the development of antiamyloid drugs. Several compounds have been identified that either slow or halt the progression of fibril formation, and they are presumed

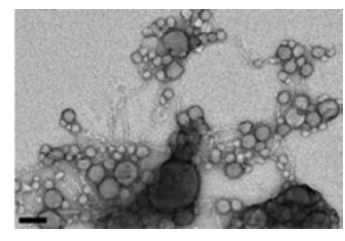
to act via discrete, ligand-like binding to the proteins. Feng et al. now report that these compounds are more likely acting via nonspecific mechanisms. They showed that several of these inhibitors form large aggregates that can encapsulate their protein target. A parallel investigation of compounds previously known to form aggregates demonstrated that these compounds are all effective amyloid inhibitors. The activity of both sets of compounds can be ameliorated by the addition of bovine serum albumin, which is known to disrupt compound aggregates. This result may require a rethinking of drug discovery approaches for amyloid-based disorders. [Brief Communications, p. 197; News \& Views, p. 159]

\section{Sulfation determination}

Heparan sulfate biosynthesis requires a wealth of enzymes to assemble and decorate the core sugar chain. The 3-O-sulfotransferases introduce sulfate groups at the $\mathrm{C} 3$ hydroxyl of glucosamine residues, but their preferences for neighboring residues differs, with the result that the modified chains are structurally and biologically distinct. Xu et al. now report the crystal structure of a new sulfotransferase isoform that allows an understanding of how these enzymes identify their substrates. In particular, comparison of three isoforms demonstrated that a distal 'gate' controls the size of the ligand; as the gate residues increase in size, the ability of the enzyme to accommodate a neighboring sulfate group diminishes. Mutagenesis of these residues confirmed that they play a unique role in substrate selectivity. These results provide a step forward in understanding a complex biosynthetic pathway and may assist in biocatalysis efforts. [Brief Communications, p. 200] 